\title{
Influence of Cold Sensation on Plantar Tactile Sensation for Young Females
}

\author{
Tianyi Wang ${ }^{1, *}$, Shima Okada $^{1}$, Masaaki Makikawa $^{1}$, Masayuki Endo $^{2}$, Yuko Ohno $^{3}$ \\ ${ }^{1}$ Department of Robotics, Faculty of Science and Engineering, Ritsumeikan University, Shiga, Japan \\ ${ }^{2}$ Department of Children and Women's Health, Graduate School of Medicine, Osaka University, Osaka, Japan \\ ${ }^{3}$ Department of Mathematical Health Science, Graduate School of Medicine, Osaka University, Osaka, Japan \\ Received 14 December 2020; received in revised form 31 January 2021; accepted 04 March 2021
}

DOI: https://doi.org/10.46604/aiti.2021.6863

\begin{abstract}
Cold sensation (CS) is a cold feeling on people's hands or feet; this is a well-known health problem for young females. Plantar tactile sensation plays an important role in postural control and is affected by skin temperature. However, there is no research focusing on the relation between CS and plantar tactile sensation. In this study, we address the question of whether the CS influences plantar tactile sensation. 32 non cold sensation (Non-CS) and 31 cold sensation (CS) young females have participated in this research. A tactile sensation test was conducted at five plantar points (first and fifth toes, first and fifth metatarsal heads, and heel). Experimental results showed that although there was no significant difference at the first and fifth toes as well as the first metatarsal head and heel, the sensation threshold at the fifth metatarsal head for CS was lower than the Non-CS $(21.61 \pm 8.10 \mu \mathrm{m}, 27.42 \pm 11.02$ $\mu \mathrm{m}$ respectively, $\mathrm{p}<0.05)$. It was concluded that plantar tactile sensation for young females with cold sensation was more sensitive compared to healthy subjects.
\end{abstract}

Keywords: cold sensation, plantar sensation

\section{Introduction}

Cold sensation (CS) is frequently observed in females [1]. Previous studies reported that about $50 \%$ of women over 50 years suffered from CS [2]. Cold sensation is a cold feeling at extremities, under the environment where healthy people dose not feel cold [3]. Although CS is not perceived as a remarkable symptom [4], it has been proved that CS is not only related to higher frequency of chronic disease, but also associated to health problems during daily life [5].

Cold sensation patients frequently complain of cold on their feet. The feet are direct and often the only interface between the human body and the ground [6]. Along with the visual and vestibular systems, feedback provided from the somatosensory system at feet plays an important role in postural control [7]. The foot is able to recognize mechanical stimuli through cutaneous mechanoreceptors located in the epidermis and dermis of glabrous skin [8]. There are four types of cutaneous mechanoreceptors: Merkel's disk, Ruffini's corpuscles, Meissner corpuscles, and Pacinian corpuscles. Except for Meissner corpuscles, where the skin temperature will not affect its function, it was reported that cooling the skin reduced the cutaneous sensation and firing response of mechanoreceptors [9].

Previous research has revealed the relation between the foot sole temperature and plantar cutaneous sensation only through a passive cooling method such as cold pack [9]. It is still unclear whether CS patients have lower tactile sensation. We consider that knowledge about the relation between cold sensation and plantar tactile sensation will provide useful information for the necessary and appropriate health care for CS patients.

* Corresponding author. E-mail address: t-wang@fc.ritsumei.ac.jp 
As a consequence, the purpose of this paper is to investigate the relation between cold sensation and plantar tactile sensation. This paper consists of five parts. Section 2 describes the human subjects and the method. Results are shown at Section 3. Section 4 discusses the experimental results and Section 5 concludes this paper.

\section{Subjects and Method}

\subsection{Human subjects}

We asked sixty-three female students (Age: $21.5 \pm 1.7$ years, Height: $157.92 \pm 6.07 \mathrm{~cm}$, Weight: $51.45 \pm 6.16 \mathrm{~kg}$, Body Mass Index: $20.60 \pm 2.03 \mathrm{~kg} / \mathrm{m}^{2}$ ) with regular menstrual cycles to participate in the experiment. No subject reported any history of endocrinopathy, cardiovascular disease, gynecological conditions, or connective tissue disease. Subjects were required to abstain from alcohol and caffeine for at least one day and from any food at least $2 \mathrm{~h}$ before the experiment.

Before the experiment, all subjects were informed of the purpose and process of this study and provided written informed consent. Subjects were free to withdraw from the study at any time. This study was approved by the Ethics Committee on Osaka University Hospital (No. 19162, August 2019). The subjects with cold sensation was defined according to our previous study [10].

\subsection{Experiment environment}

The experiment was performed from 2019 July 31 to 2019 October 21, 10:00 to 16:00. The experiment was only conducted during follicular phase; taking consideration about the body temperature fluctuates depending on the phase of the menstrual cycle. Local temperature during the experiment was from $30.1 \pm 12.3{ }^{\circ} \mathrm{C}$ to $22.8 \pm 4.7{ }^{\circ} \mathrm{C}$, average $26.9 \pm 4.4{ }^{\circ} \mathrm{C}[11]$. The indoor temperature was $24.6 \pm 0.6{ }^{\circ} \mathrm{C}$, humidity was $54.5 \pm 12.3 \%$.

\subsection{Plantar cutaneous sensation test}

Tsuneya et al. [12] reported that insufficient toe contact, also known as floating toe, was observed in $76.2 \%$ of females and particularly on the fifth toe. As insufficient toe contact could reduce experiment accuracy, before the plantar cutaneous sensation test, we first perform a foot pressure distribution test to check whether subjects' plantar could fully contact the ground.

Fig. 1 describes the method and example of the foot pressure distribution test. Fig. 1(a) shows the overview of foot pressure measurement. I-SCAN150@ NITTA pressure sensor sheet $(165 \times 165 \times 0.1 \mathrm{~mm})$ was used to measure the subjects' dominant feet. This sensor sheet contains 1,936 sensor points, and the high resolution could satisfy the need. Fig. 1(b) described two types of foot pressure distributions. In this study, if the subject's toe was well contacted, it was defined normal; if any toe was not well contacted, it was defined floating toe and we did not test the plantar tactile sensation at the toe.

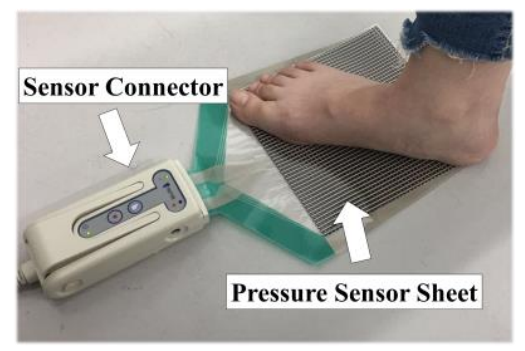

(a) Method for measuring foot pressure distribution
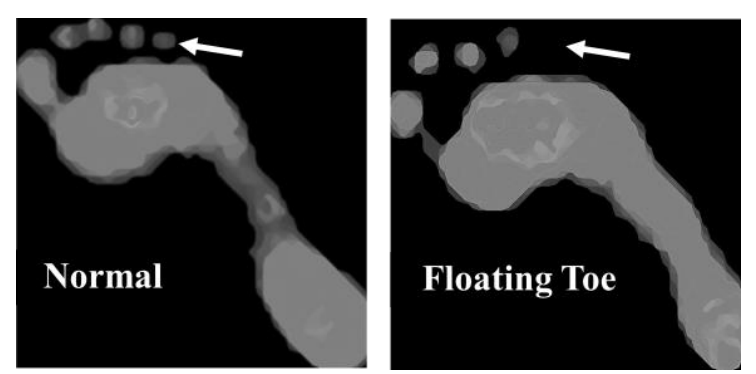

(b) Left: normal foot pressure distribution; Right: fifth toe floating

Fig. 1 Foot pressure distribution test

Fig. 2 shows an overview of the plantar tactile sensation test. Fig. 2(a) shows the system overview of plantar tactile sensation testy system. In this study, @ASUKA ELECTRI, a plantar tactile stimulation platform $(400 \times 400 \times 75 \mathrm{~mm})$ was 
utilized. This system mainly comprises three parts: a sensation stimulation platform, a response button, and a computer for motor control and data processing. Fig. 2(b) showed the mechanism of plantar tactile stimulation. The contact part is driven using a miniature linear guide with a motor-ball screw system, which can provide variable ranges from 5 to $2000 \mu \mathrm{m}$ and velocity of shearing movement from 0.1 to $250 \mathrm{~mm} / \mathrm{s}$ [13].

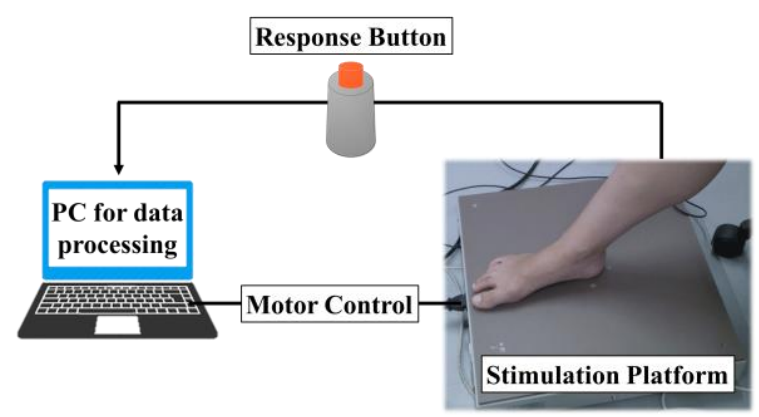

(a) System overview of plantar tactile sensation test system

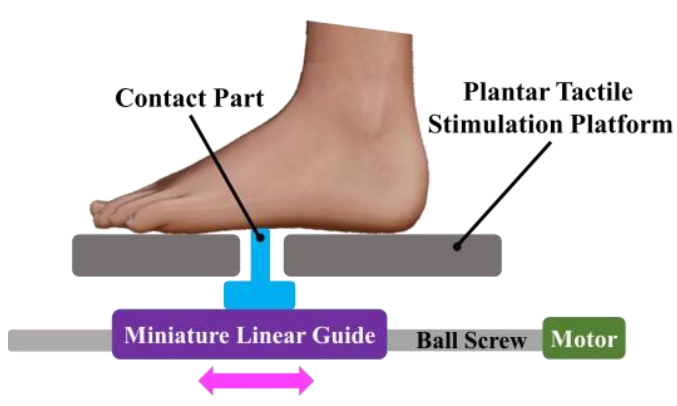

(b) Mechanism of plantar tactile stimulation

Fig. 2 Plantar tactile sensation test system

Plantar sensation test points are shown in Fig. 3(a). In this research, five plantar points were selected as test points. To eliminate serial effects, the stimulation order was set as 1: first toe, 2: heel, 3: fifth toe, 4: first metatarsal head, 5: fifth metatarsal head [14]. White circles denote the test points, and the dotted line with a single arrow denotes the stimuli order. Fig. 3(b) describes how to define the sensation threshold. First row denotes the stimulation level; second row shows the subject's feeling at the feet; third row shows the response to stimulation. Twenty stimulation levels were set for the experiment. The subjects were told to press the response button as fast as they can after they felt the movement of the contact part. If they felt the movements and continuously pressed the response button three times, stimulation at the first time when the response button was pressed was defined as the threshold at that plantar point. All tests were conducted on the subjects' dominant feet.

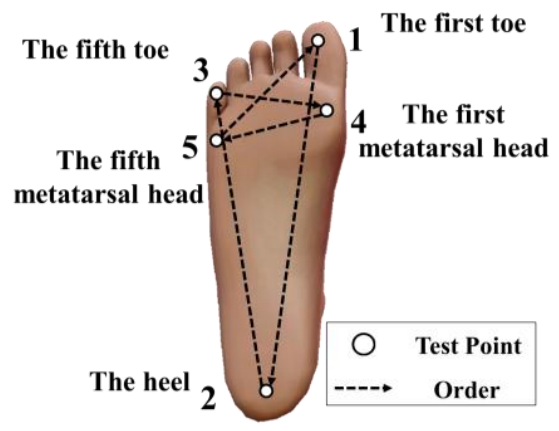

(a) Foot map with test points and stimuli order

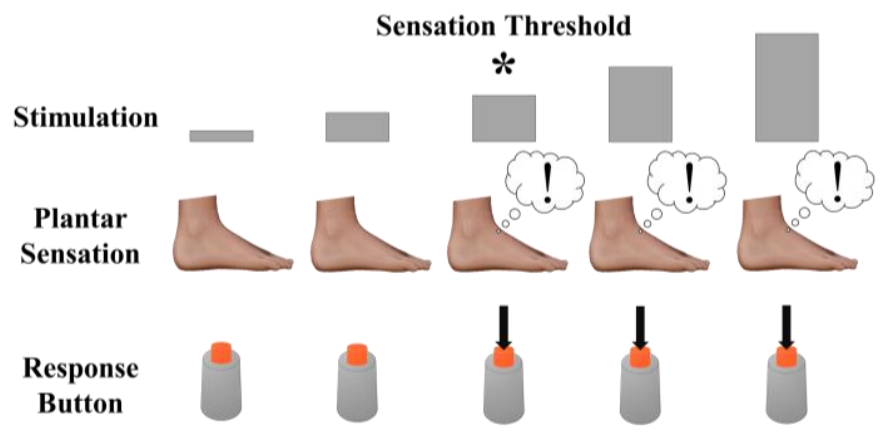

(b) Method for defining sensation threshold

Fig. 3 Test points and sensation threshold

Table 1 Threshold, stimulation time, and frequency for each stimulation level.

\begin{tabular}{ccccccccccc}
\hline \hline Level & 1 & 2 & 3 & 4 & 5 & 6 & 7 & 8 & 9 & 10 \\
\hline Threshold $(\mu \mathrm{m})$ & 5 & 10 & 15 & 20 & 25 & 30 & 35 & 40 & 45 & 50 \\
Time $(\mathrm{msec})$ & 2 & 4 & 6 & 8 & 10 & 12 & 14 & 16 & 18 & 20 \\
Frequency $(\mathrm{Hz})$ & 500 & 250 & 167 & 125 & 100 & 83 & 71 & 63 & 56 & 50 \\
\hline \hline Level & 11 & 12 & 13 & 14 & 15 & 16 & 17 & 18 & 19 & 20 \\
\hline Threshold $(\mu \mathrm{m})$ & 55 & 60 & 65 & 70 & 75 & 80 & 85 & 90 & 95 & 100 \\
Time $(\mathrm{msec})$ & 22 & 24 & 26 & 28 & 30 & 32 & 34 & 36 & 38 & 40 \\
Frequency $(\mathrm{Hz})$ & 45 & 40 & 38 & 36 & 33 & 31 & 29 & 28 & 26 & 25 \\
\hline \hline
\end{tabular}

Table 1 shows details of the twenty stimulation levels. Stimulation range (Threshold) was set as $5 \mu \mathrm{m}, 10 \mu \mathrm{m}, 15 \mu \mathrm{m}, \ldots$, $90 \mu \mathrm{m}, 95 \mu \mathrm{m}, 100 \mu \mathrm{m}$, with the same velocity of $5 \mathrm{~mm} / \mathrm{s}$. Thus, stimulation time and frequency can be calculated as following: 


\subsection{Data analysis}

Plantar tactile threshold data were obtained from a special software ("Stimulation Threshold" (CASUKA ELECTRI). The Student's t-test was used to test the significance of difference between two groups, with the significance level set at $5 \%$. The t-test was performed using MATLAB R2020a @MathWorks.

\section{Results}

The subjects' basic information is summarized in Table 2. Bar charts in Fig. 4 show the results of plantar tactile sensation. Left part is the result of sensation threshold, and the right part is the result of response frequency. The horizontal axis shows the amplitude of threshold and frequency, and the vertical axis represents different test points. Gray bars represent the non-CS group, and white bars indicate the results of the CS group. Notice that the number of test points at the fifth toe (Point 3) was different from others. After checking the foot pressure distribution, the floating toe at fifth toe was observed in 9 subjects in the non-CS group and 14 subjects in the CS group. Thus, only 23 non-CS subjects and 17 CS subjects had the plantar sensation of fifth toe (Point 3). Although there was no significant difference of stimulation threshold at first toe, heel, fifth toe or first metatarsal head $(\mathrm{p}>0.05)$, the threshold at the fifth metatarsal head (Point 5) for the CS group was significantly lower than the non-CS group $(21.61 \pm 8.10 \mu \mathrm{m}, 27.42 \pm 11.02 \mu \mathrm{m}$, respectively, $\mathrm{p}<0.05)$. As regards to stimulation frequency, the metatarsal parts (Points 4 and 5) for the CS group showed higher amplitudes than the non-CS group (p < 0.05), $112.94 \pm 45.64 \mathrm{~Hz}$ and $90.87 \pm 32.61 \mathrm{~Hz}$ at Point $4,127.44 \pm 35.02 \mathrm{~Hz}$ and $106.80 \pm 44.80 \mathrm{~Hz}$ at Point 5 .

Table 2 Age, physical characteristics of subjects.

\begin{tabular}{cccc}
\hline \hline & $\begin{array}{c}\text { non-CS } \\
(\mathrm{N}=32)\end{array}$ & $\begin{array}{c}\mathrm{CS} \\
(\mathrm{N}=31)\end{array}$ & $\mathrm{p}$ value \\
\hline Age (years) & $21.09 \pm 1.80$ & $21.97 \pm 1.52$ & $<0.05$ \\
Height $(\mathrm{cm})$ & $158.70 \pm 6.28$ & $157.11 \pm 5.84$ & 0.30 \\
Weight $(\mathrm{kg})$ & $52.39 \pm 7.04$ & $50.47 \pm 5.03$ & 0.22 \\
BMI $\left(\mathrm{kg} / \mathrm{m}^{2}\right)$ & $20.75 \pm 2.01$ & $20.48 \pm 2.08$ & 0.60 \\
\hline \hline
\end{tabular}

Value: mean \pm standard deviation

BMI: Body Mass Index

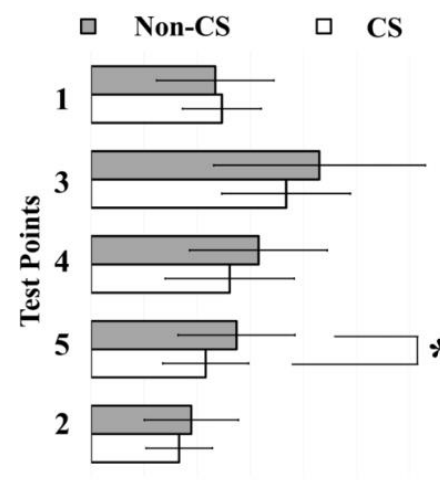

$\begin{array}{llllllll}0 & 10 & 20 & 30 & 40 & 50 & 60 & 70\end{array}$ Threshold $[\mu \mathrm{m}]$ N(Non-CS $)=32$
$N(C S)=31$

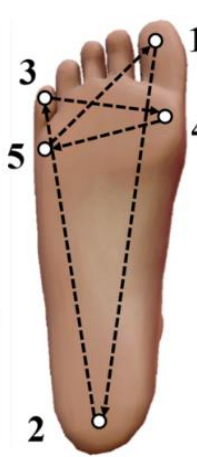
$\square$ Non-CS $\square$ CS
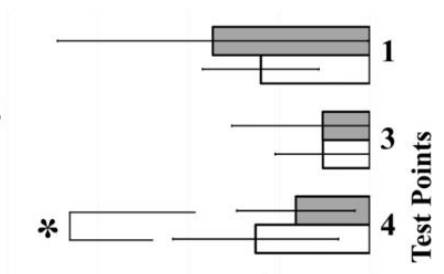

$*$
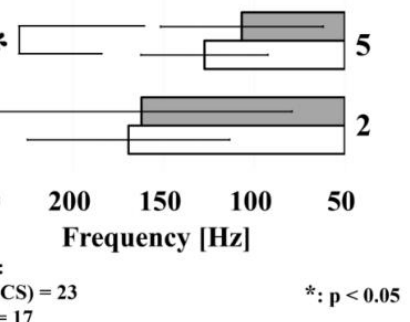

Point 3: $\mathrm{N}($ Non-CS $)=23$ $\mathrm{N}(\mathrm{CS})=17$

Fig. 4 Result of threshold and frequency to plantar tactile sensation

Fig. 5 shows the result of the threshold-frequency plot of the plantar sensation and response ranges for three mechanoreceptors. The blue square represents the Meissner corpuscle, the orange square represents the Pacinian corpuscle, and the green square represents the Ruffini ending. Markers in red and blue represent the data of the non-CS and CS groups, respectively. The dashed line is the characteristic curve of threshold-frequency and plotted using the data shown in Table 1. Standard deviations for the threshold and frequency were plotted in black solid lines. The frequency range of the Meissner corpuscle was 3-100 Hz, Ruffini ending was 15-400 Hz, and Pacinian corpuscle was 10-500 Hz [15]. 


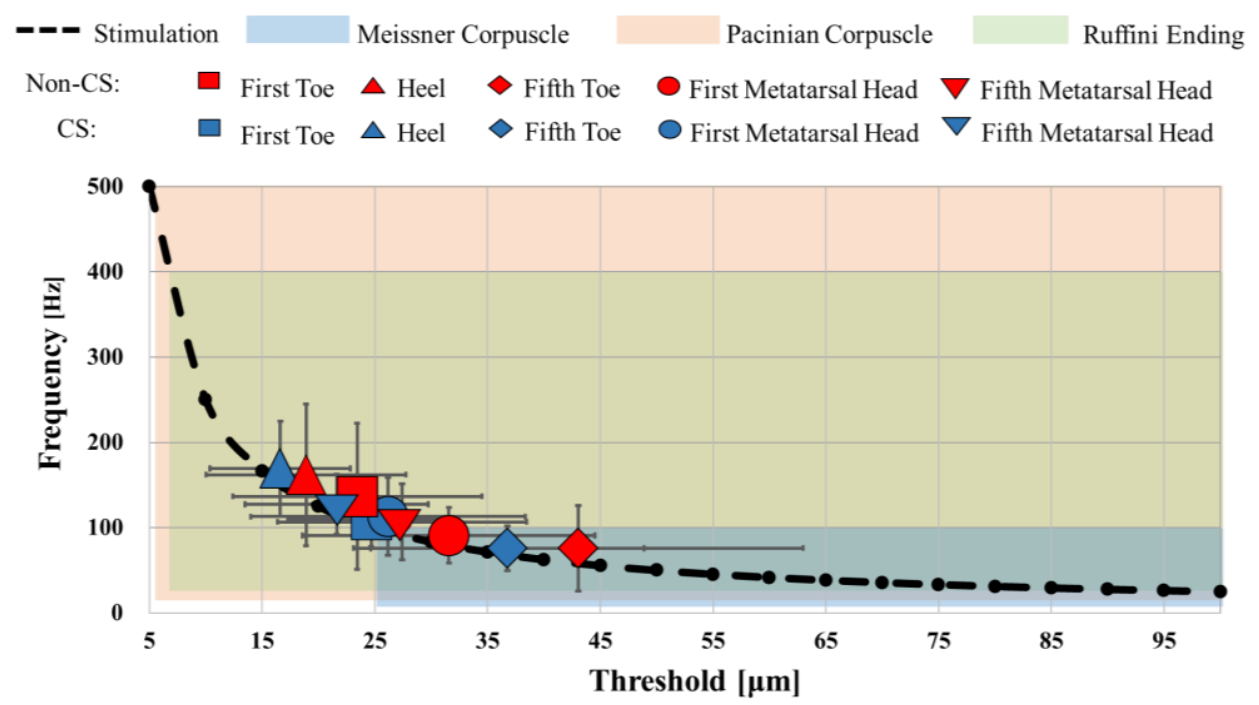

Fig. 5 Threshold-Frequency of plantar tactile sensation for the non-CS and CS groups

\section{Discussion}

According to the results of the tactile sensation test, the threshold at the fifth toe (Point 3) was higher than the other four points both for CS and non-CS groups. Ino et al. [14] tested the plantar sensation at the same points in this research and observed that the fifth toe had the highest plantar detection threshold, followed by the first metatarsal head. Surprisingly, we observed that young females with CS had a lower tactile threshold at the foot sole, opposite to the findings in the previous study. In [2], we reported that plantar sensation thresholds were higher for the CS group $(n=18)$ than the non-CS group ( $=11)$ and that young females with CS were less sensitive at the foot than the healthy subjects. However, the definition of CS in the previous study was completely different in [2]. We used a cooling recovery test to check whether subjects' plantar temperature could return to a particular level after cooing the feet with $15^{\circ} \mathrm{C}$ cold water. Although cold water stimulation has been proved as an effective approach to defining CS, we did not crosscheck the result with either the questionnaire or the thermal check methods. Thus, we considered that subjects diagnosed with CS in the previous study were different from the CS group in this research.

As previously mentioned, there are four types of mechanoreceptors distributed on the plantar glabrous skin. They are physiologically classified according to their adaption characteristics and the size of the receptive fields [16]: Merkel's disk, which are slow adapting receptors with small receptive fields (SA I ), generally provide information on indentation and press; Ruffini's corpuscles, which are slow adapting receptors with large receptive fields (SA II), can detect skin stretching; Meissner corpuscles, which are fast-adapting receptors with small receptive fields (FA I ), is more responsive to tactile events such as the motion or slippage of an object across the skin; Pacinian corpuscles, which are faster adapting receptors with large receptive fields (FA $I$ ) , is well known as a mechanoreceptors responding to vibration. According to the result shown in Fig. 5 , all markers were located at the threshold--frequency response areas of Pacinian corpuscles and Ruffini endings (orange and green squares). Only the markers representing the fifth toes (rhombus in blue and red) and the first metatarsal head of the non-CS (red circle) group were located at the areas of all three mechanoreceptors. According to the movement of the contact part, mechanical stimulation herein involved a multi-sensory approach, including skin motion, stretch, and vibration. Thus, it is reasonable to consider that FA I , FA $\Pi$, and SA $\Pi$ mechanoreceptors participated in the role of sensing the tiny movement of the contact part.

In a 2002 study, Shimojo et al. outlined that Meissner corpuscles receive no effect from skin temperature [17]. Despite the receptive fields of Pacinian corpuscles being the largest among these four mechanoreceptors, FA $\Pi$ only takes up $12 \%$ of all foot sole cutaneous mechanoreceptors (43 in 364) [18], and the distribution of FA II along the foot sole is lower than those for 
other mechanoreceptors at the same sole area [6]. Above all, fast-adapting mechanoreceptors have little influence on plantar tactile sensation for the CS group herein. Strzalkowski et al. [19] reported that there were 74 SA II cutaneous mechanoreceptors among all 364 feet sole cutaneous mechanoreceptors, which was higher than SA I (63) and FA II (43). Moreover, according to Viseux's work [6], the percentage distribution of SA II at the metatarsal head was higher than that at the toes and the heel. Compared with skin motion and vibration, the metatarsal head seems to be more sensitive to skin stretching. In contrast, it was noticed that lowering the skin temperature enhanced the impulse firing of Ruffini endings [18]. Thus, it was suspected that the low foot skin temperature for the CS group increased the impulse firing of SA II mechanoreceptors, thereby improving foot tactile sensation.

The mechanical properties of the skin may also partially explain some difference in sensation threshold between the CS and the non-CS groups. CS patients had higher skin hardness at the foot sole [20]; compared with the toes and the heel, the highest skin hardness was observed at the fifth metatarsal head [21]. Meanwhile, it was hypothesized that when skin hardness increases, it was easier to have stress concentration and increased sensation at the finger [22].Thus, another possible reason to explain the finding in this study is: for young females with CS, lower plantar sensation threshold, namely more sensitivity at the foot, is possibly due to the higher skin hardness at the fifth metatarsal head.

The ability to detect tiny movements such as slippage varies if the stimuli direction changes [17]. Specifically, the threshold of detecting slippage increases from the vertical to the horizontal direction. In this research, all subjects were asked to put their feet on the test platform along the same direction of contact part movement. Thus, discussing the effect of stimulation direction on the sensation threshold was difficult. We will investigate this aspect in a future study. Another current technical problem is that controlling the surface temperature of the sensation test platform is difficult. A difference in temperature between the subject's foot sole and contact area may influence the test result. Although no subject reported a cold feeling when they put her foot on the test platform during the experiment, the method of avoiding cooling the foot from the experiment equipment should be focused on in a future study.

\section{Conclusions}

In this paper, plantar tactile sensation for young females with cold sensation was studied. The experiment results demonstrated that the sensation threshold at the fifth metatarsal head for the cold sensation group was lower than the non-cold sensation group. Enhanced impulse firing due to the lower skin temperature and higher skin hardness were considered the reasons for young females with cold sensation being more sensitive.

\section{Conflicts of Interest}

The authors declare no conflict of interest.

\section{References}

[1] Y. M. Hur, J. H. Chae, K. W. Chung, J. J. Kim, H. U. Jeong, J. W. Kim, et al., "Feeling of Cold Hands and Feet is a Highly Heritable Phenotype," Twin Research and Human Genetics, vol. 15, no. 2, pp. 166-169, April 2012.

[2] T. Wang, M. Endo, H. Uehara, S. Suga, M. Matsuzaki, G. Nakamoto, et al., "Plantar Tactile Sensation for Young Females with Hiesho," IEEE $2^{\text {nd }}$ Global Conference on Life Sciences and Technologies, IEEE Press, March 2020, pp. 329-330.

[3] H. Mori, H. Kuge, S. Sakaguchi, T. H. Tanaka, and J. Miyazaki, "Determination of Symptoms Associated with Hiesho among Young Females Using Hie Rating Surveys,” Journal of Integrative Medicine, vol. 16, no. 1, pp. 34-38, January 2018.

[4] S. Tsuboi, T. Mine, Y. Tomioka, S. Shiraishi, F. Fukushima, and T. Ikaga, “Are Cold Extremities an Issue in Women's Health? Epidemiological Evaluation of Cold Extremities among Japanese Women,” International Journal of Women's Health, vol. 11, pp. 31-39, January 2019. 
[5] K. H. Bae, H. Y. Go, K. H. Park, I. Ahn, Y. Yoon, and S. Lee, "The Association between Cold Hypersensitivity in the Hands and Feet and Chronic Disease: Results of a Multicentre Study," BMC Complementary Alternative Medicine, vol. 18, no. 1, pp. 1-8, January 2018.

[6] F. J. F. Viseux, "The Sensory Role of the Sole of the Foot: Review and Update on Clinical Perspectives," Neurophysiolgie Clinique, vol. 50, no. 1, pp. 55-68, February 2020.

[7] A. Kavounoudias, R. Roll, and J. P. Roll, "Specific Whole-Body Shifts Induced by Frequency-Modulated Vibrations of Human Plantar Soles,” Neuroscience Letters, vol. 266, no. 3, pp. 181-184, May 1999.

[8] S. D. Perry, W. E. McIlroy, and B. E. Maki, "The Role of Plantar Cutaneous Mechanoreceptors in the Control of Compensatory Stepping Reactions Evoked by Unpredictable, Multi-Directional Perturbation,” Brain Research, vol. 877 , no. 2, pp. 401-406, September 2000.

[9] C. R. Lowrey, N. D. J. Strzalkowski, and L. R. Bent, "Cooling Reduces the Cutaneous Afferent Firing Response to Vibratory Stimuli in Glabrous Skin of the Human Foot Sole," Journal of Neurophysiology, vol. 109, no. 3, pp. 839-850, February 2013.

[10] T. Y. Wang, S. Okada, M. Endo, M. Makikawa, and Y. Ohno, "Determination of Hiesho among Young Japanese Females Using Thermographic Technique,” Advanced Biomedical Engineering, vol. 10, pp. 11-17, 2021.

[11] Japan Weather Association, “Osaka Weather,” https://tenki.jp/past/2019/07/weather/6/30/47772/, June 05, 2020.

[12] M. Tsuneya and N. Usui, “Actual State of Toe Contact in the Upright Position among Healthy Adults," Phys Ther Japan, vol. 33, no. 1, pp. 30-37, 2006. (In Japanese)

[13] M. Sato, N. Takahashi, S. Yoshimura, K. Yamashita, C. Wada, S. Ino, et al., "A New Device for Foot Sensory Examination Employing Auto-Presentation of Shear Force Stimuli Against the Skin," Journal of Biomechanical Science and Engineering, vol. 10, no. 2, pp. 1-11, April 2015.

[14] S. Ino, M. Chikai, N. Takahashi, T. Ohnishi, K. Doi, and K. Nunowaka, “A Pilot Study of a Plantar Sensory Evaluation System for Early Screening of Diabetic Neuropathy in a Weight-Bearing Position," $36^{\text {th }}$ Annual International Conference of the IEEE Engineering in Medicine and Biology Society, IEEE Press, August 2014, pp. 3508-3511.

[15] S. Choi and K. J. Kuchenbecker, "Vibrotactile Display: Perception, Technology, and Applications," Proceedings of the IEEE, vol. 101, no. 9, pp. 2093-2104, September 2013.

[16] G. Schlee, "Quantitative Assessment of Foot Sensitivity: The Effects of Foot Sole Skin Temperature, Blood Flow at the Foot Area and Footwear," Ph.D. dissertation, Faculty of Behavioral and Social Sciences, Chemnitz University of Technology, Chemnitz, SN, 2010.

[17] M. Shimojo, "Information Processing of Skin Sensation,” Journal of the Society of Instrument and Control Engineers, vol. 41, no. 10, pp. 723-727, 2002. (In Japanese)

[18] Tokyo Medical and Dental University, "Somatosensory: Mechanoceptive Sensation," http://www.tmd.ac.jp/med/phy1/ptext/somat_1.html, June 17, 2020. (In Japanese)

[19] N. D. J. Strzalkowski, R. M. Peters, J. T. Inglis, and L. R. Bent, "Cutaneous Afferent Innervation of the Human Foot Sole: What Can We Learn from Single-Unit Recordings?” Journal of Neurophysiology, vol. 120, no. 3, pp. 1233-1246, September 2018.

[20] A. Ushiroyama, "Clinical Analysis and Treatment of Hiesho," Journal of Clinical and Experimental Medicine, vol. 215, no. 11, pp. 925-929, 2005. (In Japanese)

[21] Y. Jammes, M. Viala, W. Dutto, J. P. Weber, and R. Guieu, "Skin Hardness and Epidermal Thickness Affect the Vibration Sensitivity of the Foot Sole," Clinical Research on Foot \& Ankle, vol. 5, no. 3, pp. 1-5, August 2017.

[22] H. Jeong, M. Kaneko, M. Higashimori, and K. Matsukawa, "Improvement of Tactile Sensitivity under Pressing a Finger Base," Transactions of the Society of Instrument and Control Engineers, vol. 43, no. 11, pp. 973-979, November 2007.

Copyright $\odot$ by the authors. Licensee TAETI, Taiwan. This article is an open access article distributed under the terms and conditions of the Creative Commons Attribution (CC BY-NC) license (https://creativecommons.org/licenses/by-nc/4.0/). 